\title{
Фактор мощности твердых растворов на основе теллурида висмута в области топологических фазовых переходов при высоких давлениях
}

\author{
(C) И.В. Коробейников ${ }^{1}$, Н.В. Морозова ${ }^{1}$, Л.Н. Лукьянова ${ }^{2}$, О.А. Усов ${ }^{2}$, С.В. Овсянников ${ }^{3,4}$ \\ ${ }^{1}$ Институт фризики металлов Уральского отделения Российской академии наук, \\ 620108 Екатеринбург, Россия \\ ${ }^{2}$ Физико-технический институт им. А.Ф. Иоффе Российской академии наук, \\ 194021 Санкт-Петербург, Россия \\ ${ }^{3}$ Bayerisches Geoinstitut, Universitat Bayreuth, \\ D-95447 Bayreuth, Germany \\ ${ }^{4}$ Институт химии твердого тела Уральского отделения Российской академии наук, \\ 620219 Екатеринбург, Россия \\ E-mail: i_korobeynikov@mail.ru; lidia.lukyanova@mail.ioffe.ru \\ Поступила в Редакцию 7 февраля 2019 г. \\ В окончательной редакции 10 февраля 2019 г. \\ Принята к публикации 14 февраля 2019 г.
}

\begin{abstract}
Проведены исследования коэффициента Зеебека $S$ и электропроводности $\sigma$ в твердых растворах на основе теллурида висмута с замещениями атомов в подрешетках Ві и Те при давлениях до 12 ГПа при комнатной температуре. Показано, что с увеличением давления электропроводность возрастает и, несмотря на уменьшение коэффициента Зеебека, наблюдается рост фактора мощности $S^{2} \sigma$ в твердых растворах $p-\mathrm{Bi}_{0.5} \mathrm{Sb}_{1.5} \mathrm{Te}_{3}$ и $n-\mathrm{Bi}_{2} \mathrm{Te}_{1.65} \mathrm{Se}_{0.65} \mathrm{~S}_{0.7}$. Максимальное увеличение фактора мощности получено для состава $n$-Bi $\mathrm{Te}_{1.65} \mathrm{Se}_{0.65} \mathrm{~S}_{0.7}$ в области давлений 3-4 ГПа, соответствующих электронному топологическому фазовому переходу. Исследованные твердые растворы были использованы в модели термоэлектрического модуля с регулируемым механическим усилием, приложенным к термоэлементам.
\end{abstract}

DOI: $10.21883 /$ FTP.2019.06.47719.28

\section{1. Введение}

Твердые растворы халькогенидов висмута и сурьмы являются традиционными низкотемпературными термоэлектриками, эффективными для интервала температур 100-500 К при оптимальных составах и концентрациях носителей заряда. Эти материалы являются сильными трехмерными (3D) топологическими изоляторами, для которых характерна сильная связь между спином и импульсом, и обладают нетривиальной зонной структурой, линейной дисперсией и топологически защищенными поверхностными состояниями фермионов Дирака благодаря сильному спин-орбитальному взаимодействию [1-4].

Влияние топологических поверхностных состояний (ТПС) на термоэлектрические свойства материалов на основе теллурида висмута при нормальных условиях рассматривалось в работах [5-7], результаты которых указывают на перспективность этих исследований для термоэлектричества.

Одним из эффективных методов исследований топологических свойств материалов и их связи с кристаллической структурой вещества является метод внешнего давления в области электронных топологических фазовых переходов (ТФП). Анализ рамановского рассеяния, транспортных и термоэлектрических свойств при высоких давлениях в теллуриде висмута и некоторых твердых растворах показал перспективность использо- вания давления в области ТФП для повышения фактора мощности термоэлектриков за счет увеличения вклада топологических поверхностных состояний фермионов Дирака [8-11].

В работе проведены исследования коэффициента Зеебека $S$ и электропроводности $\sigma$ в области давлений $P$ до 12 ГПа при комнатной температуре в термоэлектриках $p$ - $\mathrm{Bi}_{2-x} \mathrm{Sb}_{x} \mathrm{Te}_{3}$ и $n-\mathrm{Bi}_{2} \mathrm{Te}_{3-y} \mathrm{Se}_{y}$ с замещениями атомов в одной из подрешеток Ві или Те и в многокомпонентных твердых растворах $n-\mathrm{Bi}_{2-x} \mathrm{Sb}_{x} \mathrm{Te}_{3-y} \mathrm{Se}_{y}$ $n-\mathrm{Bi}_{2-x} \mathrm{Sb}_{x} \mathrm{Te}_{3-y-z} \mathrm{Se}_{y} \mathrm{~S}_{z}$ с замещениями атомов в обеих подрешетках теллурида висмута с целью определения влияния давления на фактор мощности $S^{2} \sigma$. Рассматриваемые твердые растворы были использованы в модели термоэлектрического модуля, в котором предусмотрена регулировка приложенного к термоэлементам механического напряжения для оптимизации параметра мощности [12].

\section{2. Образцы для измерений и методика эксперимента}

Твердые растворы на основе теллурида висмута были получены методом направленной кристаллизации, который дает возможность получить многокомпонентные однородные термоэлектрики с различными замещениями атомов в подрешетках основного соединения, состоящие 
из монокристаллических зерен, вытянутых вдоль оси роста кристалла, перпендикулярной кристаллографической оси $c_{3}$. Все образцы кристаллизованы в стандартной ромбоэдрической структуре $R \overline{3} m$. Слоистые микрообразцы для исследований термоэлектрических свойств под высоким давлением получали в виде сколов вдоль плоскости Ван-дер-Ваальса (0001) монокристаллических зерен, вырезанных из исходных образцов.

Измерения термоэлектрических свойств проводились в камере высокого давления с двумя наковальнями при непрерывном изменении давления на образце до 12 ГПа при комнатной температуре $[8,12]$. Камера высокого давления помещалась в компактную установку с прессом, которая позволяла постепенно увеличивать давление с автоматической регистрацией всех необходимых сигналов от образца. Величина давления внутри камеры рассчитывалась с использованием калибровочной кривой, определенной по хорошо известным и четко наблюдаемым фазовым переходам в ряде полупроводниковых материалов. В данной работе использованы два типа наковален: 1) плоские наковальни, изготовленные из синтетических алмазов с диаметром рабочей поверхности $\sim 600$ мкм, 2) полусферически вогнутые наковальни из твердосплавных материалов с рабочим диаметром $\sim 1$ мм. В камере первого типа измерялись тонкие образцы размером $200 \times 200 \times 30$ мкм, а в камера второго типа проводились измерения с образцами размером $150 \times 150 \times 150$ мкм. В обеих камерах образец помещался в контейнер из известняка (среда, передающая давление). Электросопротивление измерялось с применением квази-четырехконтактного метода. При измерении коэффициента Зеебека верхняя наковальня нагревалась для создания температурного градиента $(\Delta T)$ на образце. Остальные детали эксперимента подробно описаны в работе [13].

\section{3. Термоэлектрические свойства}

На слоистых монокристаллических образцах твердых растворов $p$ - $\mathrm{Bi}_{0.5} \mathrm{Sb}_{1.5} \mathrm{Te}_{3}, \quad n-\mathrm{Bi}_{1.6} \mathrm{Sb}_{0.4} \mathrm{Te}_{2.9} \mathrm{Se}_{0.1}$, $n-\mathrm{Bi}_{2} \mathrm{Te}_{1.65} \mathrm{Se}_{0.65} \mathrm{~S}_{0.7}$ были измерены коэффициент Зеебека $S$ (рис. 1-3, кривые 1 ) и удельная электропроводность $\sigma$ (рис. 1-3, кривые 2) в зависимости от давления $P$ при комнатной температуре. В составе $n-\mathrm{Bi}_{2} \mathrm{Te}_{2.76} \mathrm{Se}_{0.24}$ была измерена только зависимость $S(P)$ (рис. 2, кривая 4).

Для всех исследованных твердых растворов с ростом приложенного давления электропроводность $\sigma$ возрастает, а коэффициент Зеебека $S$ уменьшается (рис. 1-3). Слабое снижение коэффициента Зеебека наблюдается в твердых растворах $n$ - $\mathrm{Bi}_{2} \mathrm{Te}_{1.65} \mathrm{Se}_{0.65} \mathrm{~S}_{0.7}$ и $n-\mathrm{Bi}_{2} \mathrm{Te}_{2.76} \mathrm{Se}_{0.24}$ до давлений 6-7ГПа, а в $n-\mathrm{Bi}_{1.6} \mathrm{Sb}_{0.4} \mathrm{Te}_{2.9} \mathrm{Se}_{0.1} \quad$ и $p-\mathrm{Bi}_{0.5} \mathrm{Sb}_{1.5} \mathrm{Te}_{3}$ до $P=3-4$ ГПа, при этом логарифмические зависимости $|S|$ от $P$ остаются близкими к линейным (рис. 4). Затем с повышением давления коэффициент Зеебека резко снижается, особенно в со- ставах $n-\mathrm{Bi}_{1.6} \mathrm{Sb}_{0.4} \mathrm{Te}_{2.9} \mathrm{Se}_{0.1}$ и $n-\mathrm{Bi}_{2} \mathrm{Te}_{2.76} \mathrm{Se}_{0.24}$ (рис. 4, кривые 2,3).

На логарифмических зависимостях электропроводности от давления наблюдаются изменения наклонов кри-

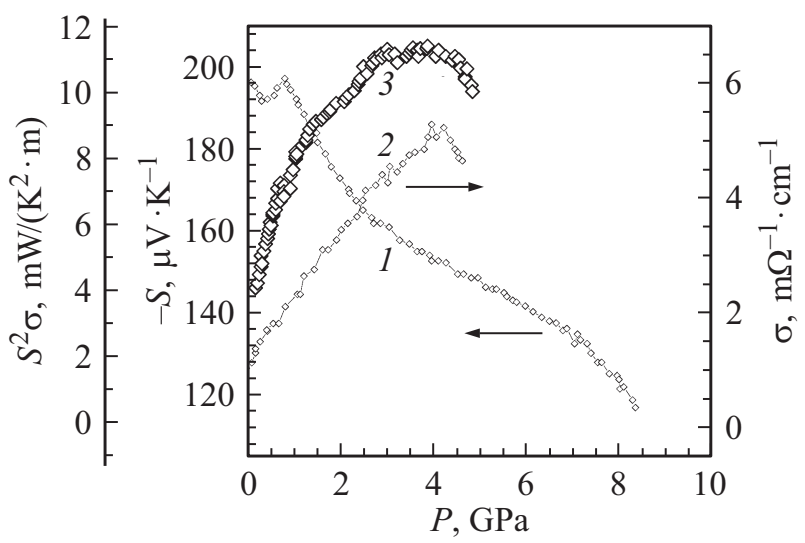

Рис. 1. Коэффициент Зеебека $S(1)$, электропроводность $\sigma(2)$ и фактор мощности $S^{2} \sigma$ в зависимости от давления в твердом растворе $n-\mathrm{Bi}_{2} \mathrm{Te}_{1.65} \mathrm{Se}_{0.65} \mathrm{~S}_{0.7}$.

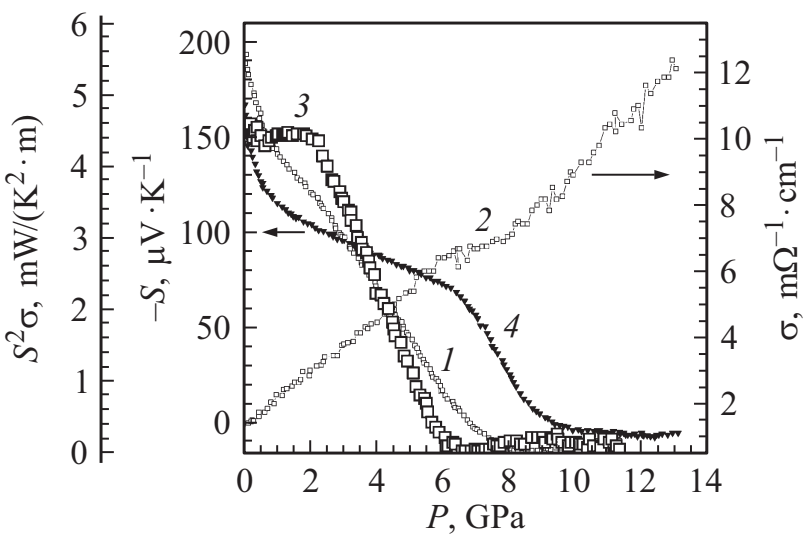

Рис. 2. Коэффициент Зеебека $S(1,4)$, электропроводность $\sigma(2)$ и фактор мощности $S^{2} \sigma(3)$ в зависимости от давления в твердых растворах $n$ - $\mathrm{Bi}_{1.6} \mathrm{Sb}_{0.4} \mathrm{Te}_{2.9} \mathrm{Se}_{0.1}$ (1-3) и $n-\mathrm{Bi}_{2} \mathrm{Te}_{2.76} \mathrm{Se}_{0.24}$ (4).

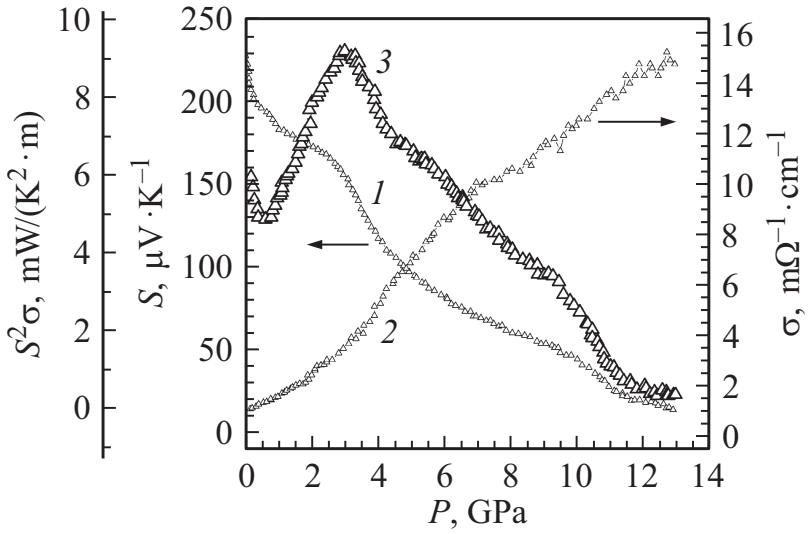

Рис. 3. Коэффициент Зеебека, $S(1)$, электропроводность $\sigma(2)$ и фактор мощности $S^{2} \sigma(3)$ в зависимости от давления в твердом растворе $p-\mathrm{Bi}_{0.5} \mathrm{Sb}_{1.5} \mathrm{Te}_{3}$. 
вых в области давлений 3-4ГПа (рис. 5). Наиболее резкий рост $\sigma(P)$ был установлен в составе $p-\mathrm{Bi}_{0.5} \mathrm{Sb}_{1.5} \mathrm{Te}_{3}$ (рис. 5, кривая 3).

Характер зависимостей коэффициента Зеебека и электропроводности определяет значительное повышение фактора мощности $S^{2} \sigma$ в твердом растворе $n-\mathrm{Bi}_{2} \mathrm{Te}_{1.65} \mathrm{Se}_{0.65} \mathrm{~S}_{0.7}$, в котором величина $S^{2} \sigma$ возрастает в 2.8 раза при $P \approx 4$ ГПа по сравнению с нормальными условиями (рис. 1, кривая 3 ) вследствие слабого снижения $S$ (рис. 1,4 , кривые 1 ) и роста $\sigma$ (рис. 1 , кривая 2 , рис. 5, кривая 1). Повышение $S^{2} \sigma$ в твердом раствоpe $p-\mathrm{Bi}_{0.5} \mathrm{Sb}_{1.5} \mathrm{Te}_{3}$ в основном связано с более резким ростом электропроводности в зависимости от давления, чем в других составах, поскольку коэффициент Зеебека в $p$ - $\mathrm{Bi}_{0.5} \mathrm{Sb}_{1.5} \mathrm{Te}_{3}$ значительно снижается при $P>4$ ГПа.

В твердом растворе $n-\mathrm{Bi}_{1.6} \mathrm{Sb}_{0.4} \mathrm{Te}_{2.9} \mathrm{Se}_{0.1}$ фактор мощности остается практически постоянным при увеличении давления до 4ГПа вследствие резкого снижения коэффициента Зеебека и недостаточного роста электропроводности, которого мало для компенсации снижения $S$ (рис. 4,5, кривые 2). При нормальных условиях этот состав является оптимальным для температур ниже комнатной, поэтому максимальные значения коэффициента Зеебека и фактора мощности в нем сдвинуты в сторону низких температур, а при комнатной температуре величины $S$ и $S^{2} \sigma$ снижаются [14]. Возможно, такой характер

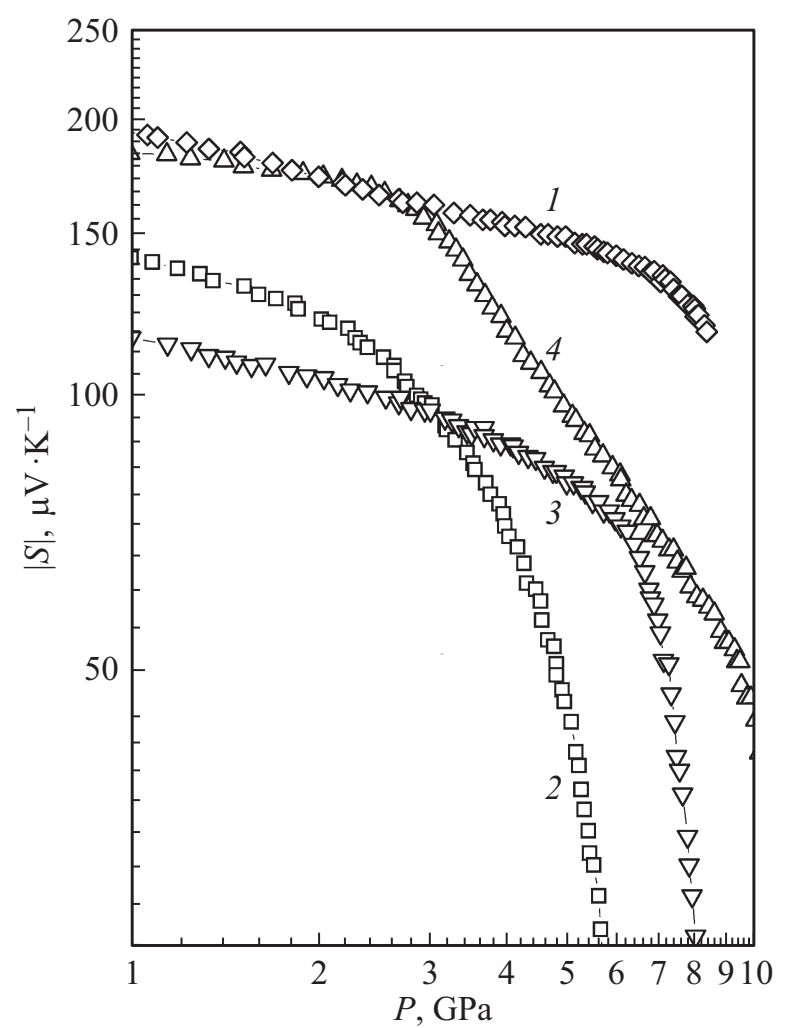

Рис. 4. Логарифмические зависимости абсолютной величины коэффициента Зеебека от давления для твердых растворов $\quad n-\mathrm{Bi}_{2} \mathrm{Te}_{1.65} \mathrm{Se}_{0.65} \mathrm{~S}_{0.7} \quad(1), \quad n-\mathrm{Bi}_{1.6} \mathrm{Sb}_{0.4} \mathrm{Te}_{2.9} \mathrm{Se}_{0.1} \quad$ (2), $n-\mathrm{Bi}_{2} \mathrm{Te}_{2.76} \mathrm{Se}_{0.24}$ (3), $p$ - $\mathrm{Bi}_{0.5} \mathrm{Sb}_{1.5} \mathrm{Te}_{3}$ (4).

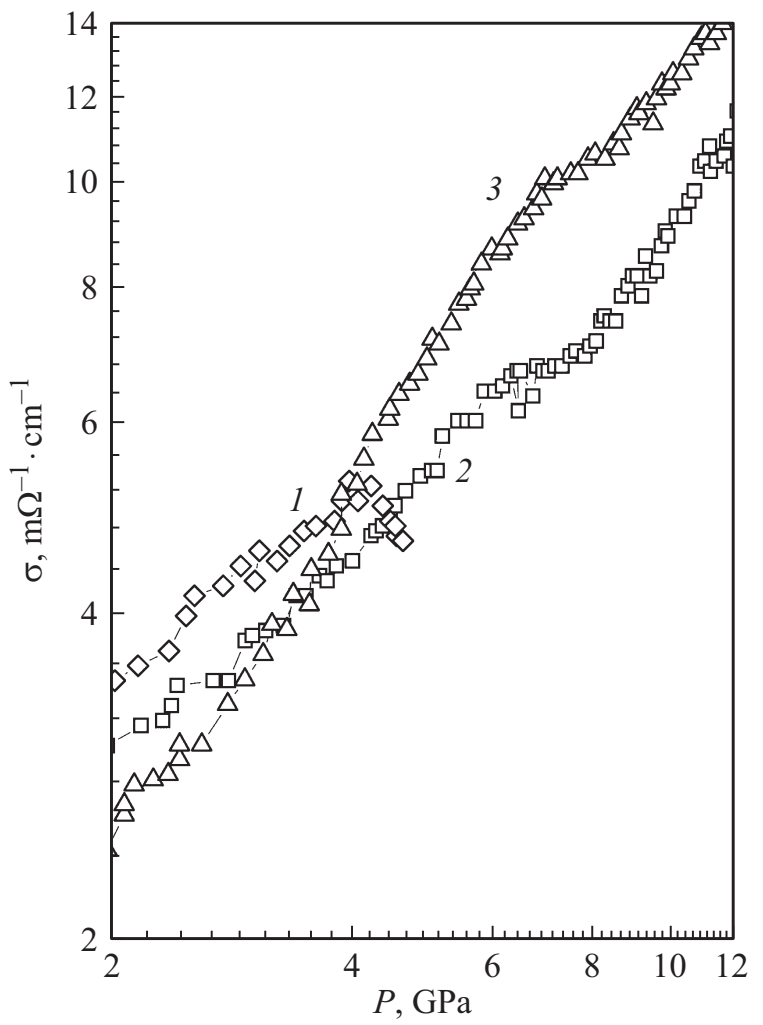

Рис. 5. Логарифмические зависимости электропроводности $\sigma$ от давления $P$ для твердых растворов $n-\mathrm{Bi}_{2} \mathrm{Te}_{1.65} \mathrm{Se}_{0.65} \mathrm{~S}_{0.7}(1)$, $n-\mathrm{Bi}_{1.6} \mathrm{Sb}_{0.4} \mathrm{Te}_{2.9} \mathrm{Se}_{0.1}$ (2), $p-\mathrm{Bi}_{0.5} \mathrm{Sb}_{1.5} \mathrm{Te}_{3}$ (3).

изменения термоэлектрических свойств в твердом растворе $n$ - $\mathrm{Bi}_{1.6} \mathrm{Sb}_{0.4} \mathrm{Te}_{2.9} \mathrm{Se}_{0.1}$ влияет на зависимость $S^{2} \sigma$ от давления.

\section{4. Анализ результатов. Топологические фазовые переходы}

В исследованных твердых растворах были обнаружены электронные изоструктурные топологические фазовые переходы $(Ф П)$ из анализа производных $d S / d P$ (рис. 6,7).

В составах $p-\mathrm{Bi}_{0.5} \mathrm{Sb}_{1.5} \mathrm{Te}_{3}$ и $n-\mathrm{Bi}_{2} \mathrm{Te}_{2.76} \mathrm{Se}_{0.24}$ с замещениями атомов в одной из подрешеток теллурида висмута топологический ФП, соответствующий минимальным значениям производной $d S / d P$, находится в области давлений 3-4ГПа (рис. 6, кривые 1,2). В $p-\mathrm{Bi}_{0.5} \mathrm{Sb}_{1.5} \mathrm{Te}_{3}$ при тех же давлениях фактор мощности достигает максимума (рис. 3, кривая 3), и происходит изменение наклона зависимости $\sigma(P)$ (рис. 5, кривая 3). Эти особенности термоэлектрических свойств и производных $d S / d P$, связанные с топологическим ФП при 3-4ГПа, сопровождаются переходом исходного сильного топологического изолятора с $Z_{2}$-инвариантом $v_{0}=1$ в слабый с инвариантом $v_{0}=0$ в результате сжатия межщелевых пространств Ван-дер-Ваальса и ослабления связи между спином и импульсом $[11,15]$. 
В многокомпонентных твердых растворах $n-\mathrm{Bi}_{2} \mathrm{Te}_{1.65} \mathrm{Se}_{0.65} \mathrm{~S}_{0.7}$ и $n-\mathrm{Bi}_{1.6} \mathrm{Sb}_{0.4} \mathrm{Te}_{2.9} \mathrm{Se}_{0.1}$ особенности на зависимостях $d S / d P$ становятся размытыми и находятся в более широкой области давлений, 2-6ГПа (рис. 7, кривые 1,2), что может быть связано с беспорядком в распределении атомов при замещениях $\mathrm{Bi} \rightarrow \mathrm{Sb}$ и $\mathrm{Te} \rightarrow \mathrm{Se}$ и изменением инверсии краев валентной зоны и зоны проводимости [10].

Особенности $d S / d P$ при более высоких давлениях, которые обычно связывают со структурными ФП, находятся вблизи 8 ГПа в твердых растворах $n$ - $\mathrm{Bi}_{2} \mathrm{Te}_{2.76} \mathrm{Se}_{0.24}$ и $n-\mathrm{Bi}_{2} \mathrm{Te}_{1.65} \mathrm{Se}_{0.65} \mathrm{~S}_{0.7}$ (рис. 6,7, кривые 2), в области $10-12$ ГПа в $n-\mathrm{Bi}_{2} \mathrm{Te}_{2.76} \mathrm{Se}_{0.24}, n-\mathrm{Bi}_{1.6} \mathrm{Sb}_{0.4} \mathrm{Te}_{2.9} \mathrm{Se}_{0.1}$ и $p-\mathrm{Bi}_{0.5} \mathrm{Sb}_{1.5} \mathrm{Te}_{3}$ (рис. 6) согласуются с $[12,10]$. Структурные ФП из ромбоэдрической фазы $R \overline{3} m$ в моноклинную $C 2 / m$ при $P=9.5$ ГПа наблюдались в твердых раствоpax $p-\mathrm{Bi}_{0.5} \mathrm{Sb}_{1.5} \mathrm{Te}_{3}[12]$ по данным исследований методом рентгеновской дифракции. В составе $\mathrm{Bi}_{1.5} \mathrm{Sb}_{0.5} \mathrm{Te}_{1.8} \mathrm{Se}_{1.2}$ при давлении 8.2 ГПа был обнаружен ФП в дираковский полуметалл [10] из исследований рентгеновской дифракции и спектров рамановского рассеяния при высоких давлениях.

Полученные значения фактора мощности (рис. 1-3, кривые 3) вместе с известными из литературы данными о теплопроводности, измеренной при давлениях

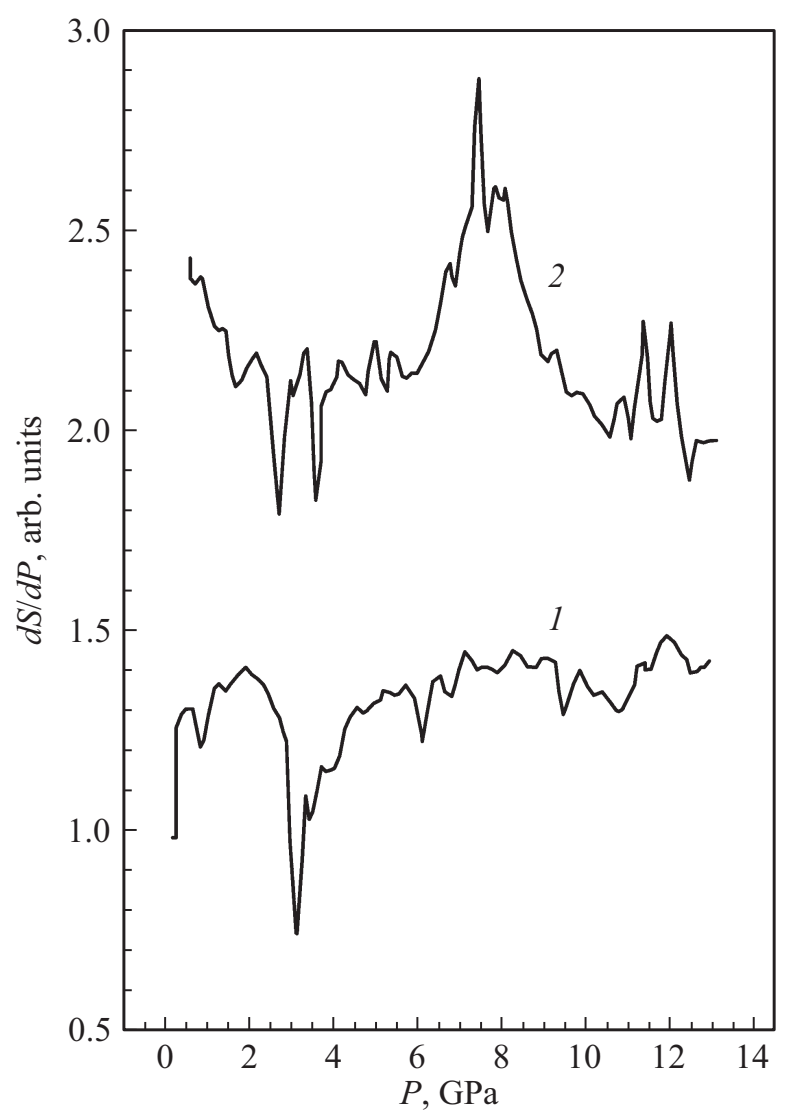

Рис. 6. Нормированные производные $d S / d P$ в зависимости от приложенного давления $P$ в твердых растворах $p-\mathrm{Bi}_{0.5} \mathrm{Sb}_{1.5} \mathrm{Te}_{3}(1), n-\mathrm{Bi}_{2} \mathrm{Te}_{2.76} \mathrm{Se}_{0.24}$ (2).

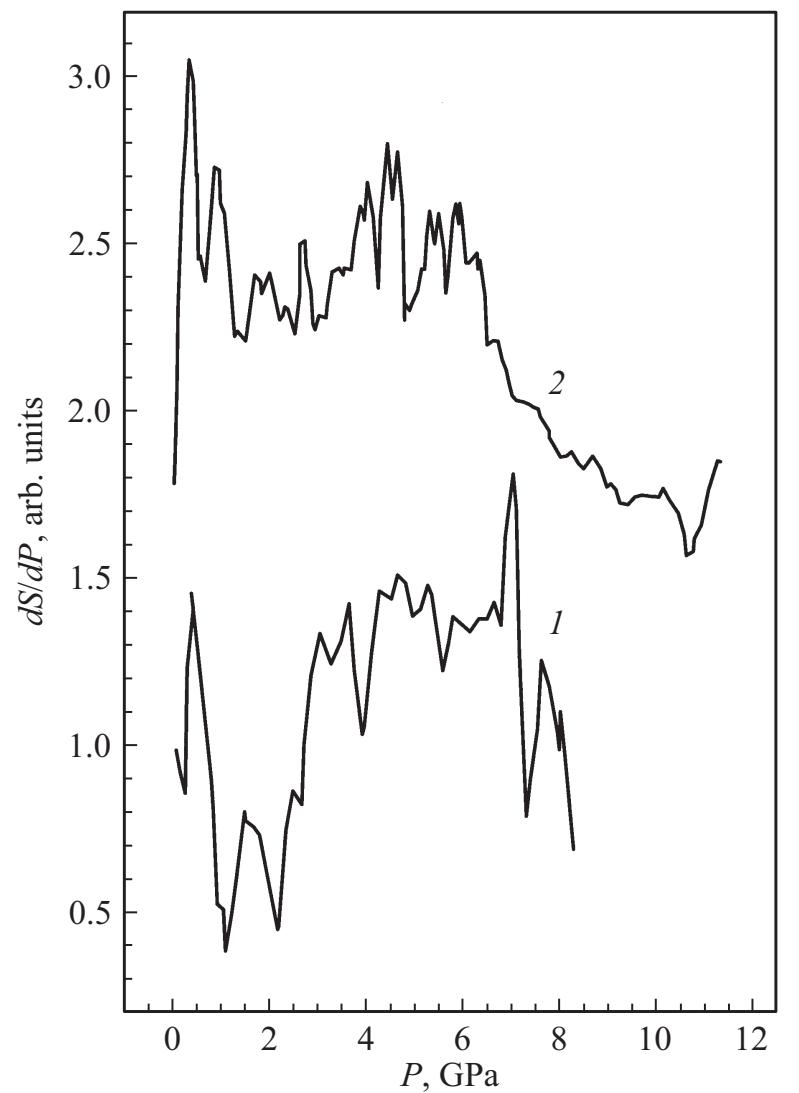

Рис. 7. Нормированные производные $d S / d P$ в зависимости от приложенного давления $P$ в твердых растворах $n-\mathrm{Bi}_{2} \mathrm{Te}_{1.65} \mathrm{Se}_{0.65} \mathrm{~S}_{0.7}(1), n-\mathrm{Bi}_{1.6} \mathrm{Sb}_{0.4} \mathrm{Te}_{2.9} \mathrm{Se}_{0.1}$ (2).

до 10 ГПа в двойных соединениях $\mathrm{Bi}_{2} \mathrm{Te}_{3}$ и $\mathrm{Sb}_{2} \mathrm{Te}_{3}$ [16], позволили провести оценки термоэлектрической эффективности. В области давлений 3-4ГПа фактор мощности достигает максимума для исследованных твердых растворов. В случае, когда теплопроводность возрастает при давлениях 3-4ГПа не более чем на 50\%, термоэлектрическая эффективность $Z$ при комнатной температуре, по предварительным оценкам, может достигать $(6-7) \cdot 10^{-3} \mathrm{~K}^{-1}$.

На основе исследованных термоэлектриков в институте Физики металлов УрО РАН была разработана модель термоэлектрического преобразователя высокого давления. В данном устройстве термоэлектрический модуль в форме диска зажат между двумя противоположными наковальнями из твердосплавных материалов. Для предотвращения механического повреждения термоэлементов модуля, а также для плавного и равномерного изменения давления внутренний объем модуля (пространство в окрестности термоэлементов) заполнен средой, передающей давление и обладающей хорошей термо- и электроизоляцией термоэлектрических элементов друг от друга в областях вне контактных точек. Камера высокого давления с одним образцом может быть рассмотрена как термоэлектрический преобразователь с одним единственным термоэлементом. Возможно несколько вари- 
антов применения такого модуля. Например, это может быть оптимизация характеристик термоэлемента при данной температуре путем регулирования величины давления или модификация давлением температурного профиля термоэлектрических параметров термоэлемента, когда конкретные термоэлектрики можно эффективно использовать в температурных диапазонах, лежащих за пределами их стандартных (при нормальном давлении) оптимальных температурных режимов, как это предложено для $\mathrm{SnSe}$ [17].

\section{5. Заключение}

Таким образом, проведенные исследования показали, что в результате приложенного давления в твердых растворах на основе теллурида висмута происходит увеличение фактора мощности в области изоструктурных фазовых топологических переходов, что делает перспективным использование разработанных термоэлектриков в термоэлектрическом модуле с регулируемым механическим напряжением.

Работа выполнена в рамках государственного задания Миниситерства образования и науки России (тема „Электрон“, № AА АА-А18-118020190098-5).

\section{Список литературы}

[1] M.Z. Hasan, C.L. Kane. Rev. Mod. Phys., 82, 3045 (2010).

[2] С.И. Веденеев. УФН, 187, 411 (2017).

[3] J. Zhang, C.-Z. Chang, Z. Zhang, J. Wen, X. Feng, K. Li, M. Liu, K. He, L. Wang, X. Chen, Q.-K. Xue, X. Ma, Y. Wang. Nature Commun., 2, 574 (2011).

[4] S.Y. Matsushita, K.K. Huynh, H. Yoshino, N.H. Tu, Y. Tanabe, K. Tanigaki. Phys. Rev. Mater., 1, 054202 (2017).

[5] T.-H. Liu, J. Zhou, M. Li, Z. Ding, Q. Song, B. Liao, L. Fu, G. Chen. Natl. Acad. Sci. USA, 115, 879 (2018).

[6] N. Xu, Y. Xu, J. Zhu. npj Quantum Materials, 2, 51 (2017).

[7] J.P. Heremans, V. Jovovic, E.S. Toberer, A. Saramat, K. Kurosaki, A. Charoenphakdee, S. Yamanaka, G.J. Snyder. Science, 321, 554 (2008).

[8] S.V. Ovsyannikov, N.V. Morozova, I.V. Korobeinikov, L.N. Lukyanova, A.Y. Manakov, A.Y. Likhacheva, A.I. Ancharov, A.P. Vokhmyanin, I.F. Berger, O.A. Usov, V.A. Kutasov, V.A. Kulbachinskii, T. Okada, V.V. Shchennikov. Appl. Phys. Lett., 106, 143901 (2015).

[9] И.В. Коробейников, Л.Н. Лукьянова, Г.В. Воронцов, В.В. Щенников, В.А. Кутасов. ФТТ, 56 (2), 263 (2014).

[10] J.-S. Kim, R. Juneja, N.P. Salke, W. Palosz, V. Swaminathan, S. Trivedi, A.K. Singh, D. Akinwande, J.-F. Lin. J. Appl. Phys., 123, 115903 (2018).

[11] M. Zhang, X. Wang, A. Rahman, Q. Zeng, D. Huang, R. Dai, Z. Wang, Z. Zhang. Appl. Phys. Lett., 112, 041907 (2018).

[12] I.V. Korobeinikov, N.V. Morozova, L.N. Lukyanova, O.A. Usov, V.A. Kulbachinskii, V.V. Shchennikov, S.V. Ovsyannikov. J. Phys. D: Appl. Phys., 51, 025501 (2018).

[13] V.V. Shchennikov, S.V. Ovsyannikov, A.Y. Manakov. J. Phys. Chem. Solids, 71, 1168 (2010).
[14] Л.Н. Лукьянова, В.А. Кутасов, П.П. Константинов. ФТТ, 50, 2143 (2008).

[15] K. Park, J.J. Heremans, V.W. Scarola, D. Minic. Phys. Rev. Lett., 105, 186801 (2010).

[16] M.K. Jacobsen. J. Phys.: Conf. Series, 73 (9), 1154 (2012).

[17] Y. Zhang, S. Hao, L.-D. Zhao, C. Wolverton, Z. Zeng. J. Mater. Chem. A, 4, 12073 (2016).

Редактор Л.В. Шаронова

\section{Power factor of bismuth telluride based solid solutions around topological phase transitions at high pressures}

\author{
I.V. Korobeinikov ${ }^{1}$, N.V. Morozova ${ }^{1}$, L.N. Lukyanova ${ }^{2}$, \\ O.A. Usov' ${ }^{2}$, S.V. Ovsyannikov ${ }^{3,4}$ \\ ${ }^{1}$ Miheev Institute of Metal Physics, \\ Ural Branch of Russian Academy of Sciences, \\ 620108 Yekaterinburg, Russia \\ 2 loffe Institute, \\ 194021 St. Petersburg, Russia \\ ${ }^{3}$ Bayerisches Geoinstitut, Universitat Bayreuth, \\ D-95447 Bayreuth, Germany \\ ${ }^{4}$ Institute for Solid State Chemistry, \\ Ural Branch of Russian Academy of Sciences, \\ 620219 Yekaterinburg, Russia
}

Abstract The Seebeck coefficient $S$ and the electrical conductivity $\sigma$ of solid solutions based on bismuth telluride with substitutions in $\mathrm{Bi}$ and Te sublattices were studied at pressures up to $12 \mathrm{GPa}$ at room temperature. It is shown that with increasing pressure, the electrical conductivity increases and, despite the decrease in the Seebeck coefficient, an increase in the power factor $S^{2} \sigma$ is observed in $p-\mathrm{Bi}_{0.5} \mathrm{Sb}_{1.5} \mathrm{Te}_{3}$ and $n-\mathrm{Bi}_{2} \mathrm{Te}_{1.65} \mathrm{Se}_{0.65} \mathrm{~S}_{0.7}$ solid solutions. The maximum increase in the power factor was found for $n-\mathrm{Bi}_{2} \mathrm{Te}_{1.65} \mathrm{Se}_{0.65} \mathrm{~S}_{0.7}$ in the pressure range of 3-4 GPa, corresponding to the electronic topological phase transition. The investigated solid solutions were used in the model of a thermoelectric module with adjustable mechanical stress applied to thermoelements. 